\title{
Flash Data Dissemination in Unstructured Peer-to-Peer Networks
}

\author{
Antonis Papadimitriou and Alex Delis \\ University of Athens, Athens, 15784, Greece \\ \{anthony, ad\}@di.uoa.gr
}

\begin{abstract}
The problem of flash data dissemination refers to spreading dynamically-created medium-sized data to all members of a large group of users. In this paper, we explore a solution to the problem of flash data dissemination in unstructured P2P networks and propose a gossip-based protocol, termed Catalogue-Gossip. Our protocol alleviates the shortcomings of prior gossip-based dissemination approaches through the introduction of an efficient catalogue exchange scheme that helps reduce unnecessary interactions among nodes in the unstructured network. We provide deterministic guarantees for the termination of the protocol and suggest optimizations concerning the order with which pieces of flash data are assembled at receiving peers. Experimental results show that Catalogue-Gossip is significantly more efficient than existing solutions when it comes to delivery of flash data.
\end{abstract}

\section{Introduction}

The problem of rapid data dissemination in peer-topeer $(P 2 P)$ networks is now recognized as the key element to effectively address many applications including acquisition of real-time weather information, announcement of traffic-conditions, publication of stock quotes and proliferation of news and emergency alerts. Sizeable groups of users often organized in $P 2 P$ overlays are now increasingly interested in acquiring dynamic information in order to make timely decisions [9]. In the aforementioned application domains, new data has to reach large numbers of users and/or customers as quickly as possible. This specialized form of dissemination is termed flash data dissemination and refers to quickly spreading dynamically-created mediumsize data to all members of a potentially large group of users. Flash dissemination is very different from rudimentary file transfer approaches as the core requirement is that all participants have to obtain dynamic data fast and that the originator of the information might be any node in the $P 2 P$-network. Occasionally, flash data dissemination has to proceed under adverse conditions in which networks suffer from frequent node node arrivals/departures or feature nodes prone to failure with high probability during unexpected events. Such conditions appear when either emergency situations are in development or time-critical operations are involved including deployment of physical resources, handling of micro-payments among users of a community, and resumption of multi-player games.

In a scenario that entails fast dissemination of earthquake data, the objective is to efficiently propagate information regarding ongoing seismic activity to many sites so that data can be exploited for both scientific evaluation and for issuing necessary public alerts. Another equally important application is that of detecting heat and humidity while monitoring a forest area. The goal here would be to detect bush fires and gather information that would help design a strategy to combat them. The main challenge that these systems face is that timely data dissemination should be guaranteed even in light of catastrophic events that may damage computing equipment in multiple sites. In a multi-player game scenario, there is often a global game state that all peers should be aware of. This state information should be spread quickly by using a flash dissemination scheme, even if users constantly join or depart the game. Lately, unstructured $P 2 P$-networks are increasingly used to implement such systems $[4,12]$, due to their unique characteristic of ensuring reliable operation even in adverse network conditions with minor maintenance overhead. The main advantage of unstructured P2P-networks is that they impose no pre-fixed overlay on the participating nodes and by and large rely on random communication among peers for their operation. These two features allow for the flexible realization of the two fundamental operations -information search and dissemination- yielding an network capable of overcoming unexpected failures.

In this paper, we propose a new protocol for flash data dissemination in unstructured P2P-networks. The protocol features a novel gossip-based dissemination approach that reduces communication overheads among peers and thus, it achieves faster dissemination of data reports. Our dissemination algorithm termed Catalogue-Gossip, is based on a 
succinct structure called Catalogue that allows for efficient node interactions. Additionally, we develop a specialized termination discovery mechanism that provides deterministic guaranties for the reliability of the dissemination. We also discuss a more fault-tolerant variant of the protocol which implements a decentralized file chunk selection policy to increase resistance to failures on the originator of a disseminated resource.

\section{Related Work}

Overlay structure information is absent in unstructured $P 2 P$-networks and algorithms developed for such systems rely on random message exchanges. In this context, gossipbased protocols have been widely used in unstructured P2P-networks mainly due to their simplicity and increased fault tolerance. Moreover, results that have been obtained through probabilistic analysis point into the efficiency of gossip-based protocols to spread reports [8].

Simple-gossip has been applied in a number of fields including databases [3], network management [11] and information dissemination [5]. This protocol is initiated by a node that intends to disseminate a resource by selecting a fixed number -called fan-out- of random peers to forward the content in question. Every receiving peer does the same, unless it has already "received" the resource, in which case it ceases the forwarding. Unfortunately, simple-gossiping approaches focus on reliability and fault-tolerance and ignore the aspect of efficiency in terms of speed of dissemination. In fact, forwarding resources to randomly selected peers may significantly degrade performance, as peers may receive duplicate copies of resources. Another problem that there is always a possibility that a peer does not receive the disseminated information at all (i.e., the delivery is only probabilistic).

Crew [4] was the first gossip-based protocol which explicitly dealt with fast dissemination of data in unstructured P2P-networks. In order to avoid duplicate transfers, Crew-Gossip operates in a pull mode as it involves having all nodes eagerly request data they are missing, from random peers. To make all nodes aware of missing chunks, a phase of meta-data spreading via Simple-Gossip precedes the main dissemination process. As requesting peers know which files they need, duplicate data transfers are avoided. Nevertheless, Crew-Gossip has a weakness which degrades its performance. Specifically, in the beginning of the dissemination nearly all requests to random peers are unsuccessful, as no peer -apart from the originator- has any data resource to share. The existence of many unsuccessful messages offers ground for improvement when it comes to offering a more optimized flash data dissemination protocol. Our Catalogue-Gossip approach tackles the above shortcomings by introducing catalogue-based interactions among peers thus avoiding many redundant communications. In addition, we offer a decentralized implementation of a data chunk selection policy to increase fault-tolerance. To deal with the problem of probabilistic delivery, we suggest a mechanism to ensure deterministic delivery of resources to all peers in the network.

\section{The Catalogue-Gossip Protocol}

The key objective of the Catalogue-Gossip is to distribute content of arbitrary format and size to all peers constituting the network. Catalogue-Gossip relies on an underlying Membership Protocol [6, 7] for building up the unstructured network. A membership management protocol is responsible for creating consistent neighbor views at every peer, so that when a node selects randomly a peer from its view, it would be equivalent to randomly selecting from the entire set of network nodes. Moreover, gossip-based membership protocols are designed to deal efficiently with dynamic network conditions, so that the node views are consistent even in face of high churn or failure rate. Hence, membership protocols allow flash data dissemination approaches to focus on improving dissemination speed rather than handling dynamic aspects of the network.

With an unstructured network in place, CatalogueGossip disseminates resources by having all nodes spread a meta-data message called Catalogue to random peers in the system. A catalogue contains a list of data available for downloading from its owner. In the sections that follow, we outline the structures used to implement the protocol and explain how Catalogue-Gossip works towards achieving fast content dissemination.

\subsection{Data Structures}

To handle content of arbitrary size and format, the protocol treats every file in an uniform manner. It splits files into several parts or data chunks and disseminates each such chunk independently. A file is disseminated to a particular peer, if the peer in question has received all parts comprising the resource. The recipient peer is responsible for reconstructing the original file from its constituent components. To distinguish data chunks, the uploader of a file has to provide a unique $i d$ for each data chunk produced by using the MD5-algorithm [10].

Every peer maintains two structures which are necessary for the operation of the Catalogue-Gossip protocol as Figure 1 depicts. The first is a table containing all chunks that have been downloaded by a peer thus far. The table helps the node ascertain which chunks are still missing. To this end, the node can fetch missing parts from other peers. Moreover, the table of available chunks is used as an announcement (via the Catalogue messages) to other peers, 
TABLE of available chunks

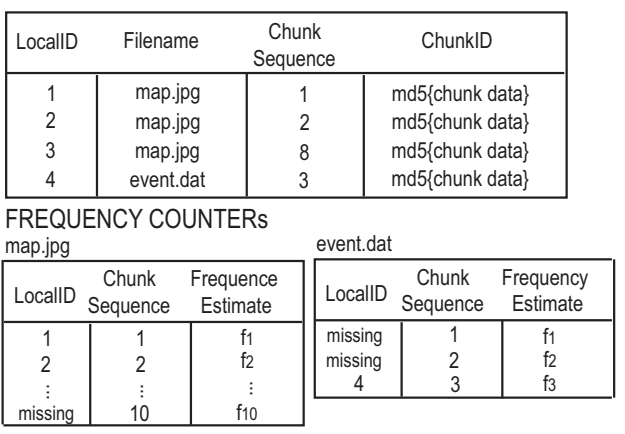

Figure 1. Catalogue-Gossip Structures

so that the latter may know which chunks are available for downloading from that specific peer. The second structure shown in Figure 1 is a set of of frequency counters whose objective is to offer an estimation on how frequent each specific chunk is in the entire $P 2 P$-system. In addition to the aforementioned two structures, Catalogue-Gossip peers trade Catalogue messages which contain information about the table of available chunks and a summary of the frequency estimates of the catalogue-sender at the moment of the Catalogue message dispatch.

The table of available chunks (Figure 1) associates each downloaded data chunk with the name of the file it belongs to and a sequence number denoting its order among the rest of the data chunks in that file. Moreover, for each separate chunk it stores the MD5 key which has been produced by feeding the contents of the particular data chunk to the message digest algorithm. Each peer's table is updated every time a new chunk is downloaded at that node, by just appending an additional record which maps to the meta-data information of the newly acquired chunk.

As mentioned above, each peer maintains a set of frequency counters shown in Figure 1. There is a frequency counter structure for every resource and/or file being disseminated in the network at any given moment. The structure in discussion helps maintain estimations of the relative frequency with which every chunk appears in the network. These estimations essentially reflect the proportion of the number of copies of each chunk to the total number of chunks of the file spread in the P2P-system. Evidently, estimations range between 0 and 1 and for each file in a peer, they sum up to 1 . If a frequency counter estimation refers to a chunk that has been already downloaded, there is a pointer to the respective record at the table of available chunks. Otherwise, even if the chunk is missing, there is still an estimation derived from trading catalogue messages with other peers.

Peers exchange messages called Catalogues during the
CATALOGUE = Frequency Counter Data + Available Chunks' IDs

\begin{tabular}{|c|c|c|c|}
\hline Filename & \multicolumn{2}{|c|}{ Frequency Estimates } & Tota \\
\hline $\begin{array}{l}\text { map.jpg } \\
\text { event.dat }\end{array}$ & \multicolumn{2}{|c|}{$\begin{array}{c}\{(1, \mathrm{f} 1),(2, \mathrm{f} 2), \ldots,(10, \mathrm{f} 10)\} \\
\{(1, \mathrm{f} 1),(2, \mathrm{f} 2),(3, \mathrm{f} 3)\}\end{array}$} & \\
\hline \multicolumn{4}{|c|}{+} \\
\hline & Filename & \multicolumn{2}{|c|}{ ChunkID } \\
\hline & $\begin{array}{l}\text { map.jpg } \\
\text { map.jpg } \\
\text { map.jpg } \\
\text { event.dat }\end{array}$ & \multicolumn{2}{|c|}{$\begin{array}{l}\text { md5\{chunk data\} } \\
\text { md5\{chunk data\} } \\
\text { md5\{chunk data\} } \\
\text { md5\{chunk data\} }\end{array}$} \\
\hline
\end{tabular}

Figure 2. The Catalogue message

whole course of dissemination. These messages (Figure 2) are a critical component for the efficient operation of the proposed dissemination scheme. Dispatched by a peer, a Catalogue contains a summary of the frequency counter data it has estimated thus far and a list with all the data chunks that the peer has available for others. Each peer that receives a catalogue can select a chunk to fetch from the list and moreover it can use the frequency data to improve its own estimations. It is worth mentioning that the contents of this Catalogue are time-dependent. The content of Catalogue changes during the dissemination, according to the chunks available by that peer at any given moment as well as the current estimation of its frequency counters.

\subsection{Basic Protocol Operation}

Every peer in Catalogue-Gossip runs four separate modules that synegistically implement the operations of the protocol. Figure 3 depicts how the components Catalogue Processing Listener, Choker, Transfer Manager and Catalogue Dispatcher cooperate. The Catalogue Processing Listener is the module which always waits for connections from other peers, so as to receive incoming catalogues and decide which chunk to possibly fetch. It operates in conjunction with the Choker module which works as a throttle mechanism used to reject connections if a node gets overloaded. The Transfer Manager is responsible for carrying out the data chunk transfers the peer is engaged in. Lastly, the Catalogue Dispatcher forwards updated versions of the Catalogue to randomly selected peers.

A node find itself in a passive state, if it does not forward its Catalogue to others. A peer in passive state may be either entirely idle simply waiting for connections or just taking part in some chunk transfers. A node is its active phase, if its Catalogue Dispatcher component has commenced gossiping the local Catalogue. The state of the node is turned into active the first time it downloads a chunk of a new file. The peer leaves its active state once the protocol has determined that the dissemination of the file is over.

Upon accepting a new connection, the Catalogue Pro- 


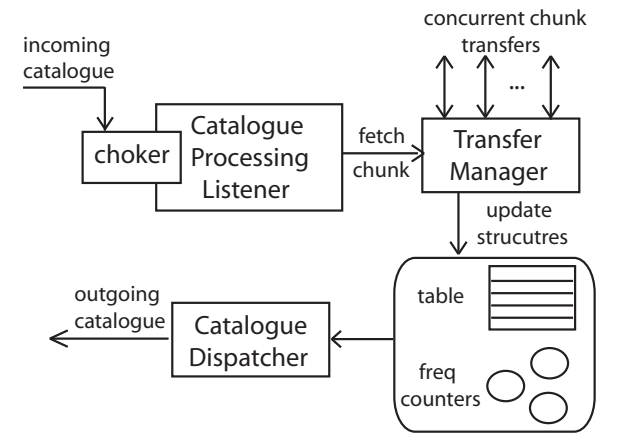

Figure 3. Logical Components of the Protocol

cessing Listener scans the incoming Catalogue to see which chunks are candidates for fetching from the remote peer. A chunk becomes a candidate for fetching, if it is contained in the incoming Catalogue, but missing from the local peer. The peer selects which chunks to fetch according to its own chunk selection policy. Once it has decided, it notifies the Transfer Manager about the chunk id and the remote peer's address, so that the transfer can be started. Li light of the fact that the Catalogue contains no chunk of interest to the receiving peer, the Catalogue Processing Listener responds with an "UNSUCCESSFUL" message.

The Choker module acts as a filter on the connections accepted by the Catalogue Processing Listener. More specifically, it computes the node's available bandwidth in order to decide whether to allow additional connections. If the peer does not have adequate spare bandwidth to accommodate another chunk transfer, the incoming connection is "choked" and a "BUSY" message is returned to the sender. The spare bandwidth is calculated by subtracting the bandwidth used by ongoing transfers from the total bandwidth of the node. The latter can be estimated by having the Transfer Manager keep track of the highest downloading bandwidth rate ever appearing during the node's operation. Because of the potentially vast number of connections established in this gossip-based environment, this measured value quickly approximates the actual maximum bandwidth of a node [4].

During the dissemination of a file, any peer can take part in multiple data chunk transfers. The Transfer Manager is a multi-threaded module able of handling concurrent connections to remote hosts. Besides that, its main responsibility is to update the structures maintained by the peer any time a chunk transfer is complete. The Transfer Manager does so by appending a record to the table of available chunks and by updating the estimates of the frequency counters. If the peer is in its passive state, the Transfer Manager initiates the Catalogue Dispatcher module to start gossiping the node's Catalogue.

As soon as a node enters its active state, the Catalogue
Dispatcher begins gossiping the local Catalogue to other peers. The node selects a peer at random from its local view of neighbors. As mentioned earlier, this is equivalent to selecting a random peer from the entire system. The node can now construct an updated version of the local Catalogue and forward it to the selected remote peer. This procedure is constantly repeated during the dissemination of a file. The peer leaves its active state when the protocol detects that the dissemination is over. A system parameter regarding the active state of a node is the inter-gossip time interval. This parameter denotes the time period elapsed between successive pushes of the Catalogue and may designate the degree of aggressiveness in the behavior of each node. Aggressive behavior as exemplified by short intervals may cause more load imbalances across nodes, with the originator suffering the highest load. This is due to the fact that the initiator may send more Catalogues during a single chunk transfer which in turn results in more peers fetching chunks from the initiator. The same holds for all the nodes that start gossiping their Catalogue early during the dissemination, thus, leading to uneven load distribution.

\subsection{Protocol Enhancements}

\subsubsection{Dissemination Termination Mechanism}

A potential disadvantage of gossip-based systems is that there are no guarantees that a disseminated resource will ultimately reach all network nodes. In [8], the fan-out (i.e. number of gossip targets) required at each node is computed, in order to achieve reliable dissemination of a resource, with very high probability. Essentially, only probabilistic guaranties can be provided in a simple gossip-based setting. Hence, an additional mechanism is required to provide deterministic guaranties. In Catalogue-Gossip, deterministic delivery is attained by having all peers continuing to forward their Catalogue until the dissemination completes. The question which emerges is how each node will know that the dissemination of a file is complete across the entire network, so that it can stop forwarding its Catalogue.

To address the issue we develop a termination mechanism. Our protocol instructs peers to leave their active phase by using a heuristic condition. Each node that forwards its Catalogue expects either the beginning of a chunk transfer or an "UNSUCCESSFUL" response. Receiving many successive unsuccessful responses is an indication that the dissemination has either concluded or is almost complete. The exact number of responses $(r)$ that would indicate the termination of the dissemination is a system parameter.

To ease the proper tuning of this parameter, we provide the following analysis. Consider a network which consists of $N$ nodes, $M$ of which have completed (i.e. received all file chunks) and $K$ are still pending. For providing the analysis we make the assumption that the dissemination pro- 
ceeds in steps. We say that a set of $K$ nodes is isolated for one round, if none of the $M$ completed nodes sends a Catalogue to any of the $K$ nodes. The probability of a one-round isolation is equal to the probability that all $M$ nodes select a completed node ${ }^{1}$ to forward the Catalogue to. So we have:

$$
\begin{gathered}
q=\operatorname{Pr}(\text { a node selects a completed node to send to }) \\
q=\frac{M}{N}=1-\frac{K}{N}
\end{gathered}
$$

and,

$$
\begin{gathered}
p=\operatorname{Pr}(\text { all } M \text { nodes select a completed node }) \\
p=q^{M}=\left(1-\frac{K}{N}\right)^{N-K}
\end{gathered}
$$

finally,

$$
\begin{gathered}
P_{r}=\operatorname{Pr}(p \text { happens in } r \text { successive rounds }) \\
P_{r}=p^{r}=\left(1-\frac{K}{N}\right)^{r(N-K)}
\end{gathered}
$$

Hence, according to the anticipated size of a system, one can adjust system parameter $r$ so that a node becomes isolated with as low $P_{r}$ as desired. A system with low $P_{r}$ is more accurate as in this case nodes keep on forwarding their catalogue, until the dissemination is actually completed. Allowing a higher $P_{r}$ means that more nodes might infer that the dissemination is over before this actually becomes true. Such a setting is suitable for systems with constraint resources (e.g., battery consumption in mobile networks). Here, it would be better for most nodes to stop expending resources while having their Catalogue forwarded and let a small portion of remaining nodes pull their missing chunks.

A node checks the heuristic condition only after it has collected all the chunks of a file to ensure that an unsuccessful response means that the remote node has completed the transfer of all the chunks. As soon as a peer has received $r$ successive unsuccessful responses, it leaves its active phase, stops forwarding its Catalogue and assumes that the dissemination is over.

To handle the extreme case of an isolation of $r$ rounds, we include a timeout mechanism in the protocol. This timer expires if no Catalogue is sent to a peer for a long time. In this case, the peer stops gossiping its catalogue and starts gossiping direct requests for the missing chunks to random peers. In other words, this mechanism actually allows a peer to unilaterally make a transition from the push to the pull model of operation, in order to deal with isolation.

\footnotetext{
${ }^{1}$ one of the $M$ nodes that have received all chunks.
}

\subsubsection{Resilience to Initiator Faults}

Gossip-based protocols are in general resilient to node faults and their performance degrades gracefully with crashing peers. However, they have an inherent weakness when it comes to resilience to faults on the initiator of a dissemination. It is crucial that the initiator can forward all the data chunks quickly, so that the entire file becomes replicated in the network as fast as possible. This is required so that the system can complete the dissemination even if the initiator fails early on in the process.

To reach this increased level of fault tolerance, we designed a chunk selection policy which favors rare chunks, so that all chunks can be replicated across the peers quickly. The other policy we investigated is having the recipient of a Catalogue select one chunk at random. More specifically the two policies we use are:

- Rarest-First: ideally, the rarest-first policy selects to fetch the chunk which is less common among the nodes of the $P 2 P$-network. This can facilitate speeding up the degree of data diffusion in the network earlier during the dissemination.

- Random-First: this policy instructs the peer to randomly fetch any of the available chunks, as announced by the Catalogue. Note that this approach does not incur the extra communication overhead of Rarest-First policy (the protocol could be implemented without frequency counters).

To implement a policy such as Rarest-First, there is a need to maintain a global view of the system to be able to tell which chunks are rare and which are more common. A centralized approach could readily achieve this by having every downloader register at the origin site which chunks it has already downloaded [2]. This is not applicable in the case of data dissemination in fully-decentralized $P 2 P$ networks, where no global state can be maintained at the initiator. We thus propose a decentralized implementation of the Rarest-First policy, so that it can be used in pure P2P environments.

Decentralized Rarest-First policy is implemented by using the frequency counter structures of Figure 1. Each peer maintains a frequency counter for every different file being currently disseminated in the system. This counter provides an estimation of the percentage of each chunk's copies relative to the total number of chunk copies belonging to the particular file in the system. The originator of a resource creates a counter which assigns the frequency $\frac{1}{L}$ to all $L$ chunks of the disseminated file.

When dispatching its Catalogue, the initiator includes the data of the frequency counter as illustrated in Figure 2. The recipient of the Catalogue selects one of the chunks based on its current local frequency counter and fetches the 
chunk. The data of the frequency counters of a peer has to be updated in two cases: when a new chunk is fetched and when it receives a frequency estimation from another peer.

The update in the first case is accomplished by increasing the Total variable of the file (Figure 2 ) by one and then adding $\frac{1}{\text { Total }}$ to the frequency of the chunk fetched. To retain the assertion that the sum of frequency estimations over the $L$ chunks of a file is equal to one $\left(\sum_{i=1}^{L} f_{i}=1\right)$, the added fraction has to be subtracted from the rest of frequency estimates. So it is split into $L-1$ equal parts, each of which is subtracted from the respective estimates.

For the second case, the recipient of the Catalogue has to compute the new estimation of frequencies by aggregating the information sent by the remote peer. To do so, we propose a weighted mean aggregator operator. To compute the respective weights it uses the "Total Chunks Counted" information shown in the Catalogue of Figure 2. This information is initially set to $L$ (the number of the chunks) by the originator and 0 by all other peers, and is updated each time a peer fetches a chunk or aggregates the estimates of other peers. Formally:

$$
f_{i}^{A}=M_{i}\left(f_{i}^{A}, f_{i}^{B}\right)=w_{A} f_{i}^{A}+w_{B} f_{i}^{B}
$$

with

$$
w_{k}=\frac{\text { Total }_{k}}{\operatorname{Total}_{A}+\text { Total }_{B}}, k=\{A, B\}
$$

where $\mathrm{A}$ is the recipient of the Catalogue, $\mathrm{B}$ is the sender and $f_{i}$ is the estimate of the frequency of the $i$-th chunk. Although intuitively Random-First and Rarest-First policies have comparable dissemination speed performance in the long term, Rarest-First is faster in replicating all data chunks in the system, whereas Random-First incurs less overhead in the network.

\section{Experimental Results}

In order to evaluate the efficiency of the CatalogueGossip, we created a simulation environment based on the JiST simulation engine [1]. JiST is a Java-based runtime environment for discrete event simulations. Our assessment included a number of protocols used for flash data dissemination. Specifically, the compared protocols were Catalogue-Gossip, Crew and Simple-Gossip. Our comparison mainly focuses on the scalability of the protocols for diverse network and file sizes while taking into account other performance aspects of the protocols.

In our evaluation, we used the following metrics: i) Completion-Time defined as the time taken for the successful update of all peers, ii) Coverage-Speed designated as the number of successfully updated peers at any point during the dissemination, iii) Data-Overhead measured as the number of unsuccessful messages and finally, $i v$ ) ChunkReplication defined as the time taken by the originator to send all chunks. In our experimentation, we vary the following key factors:

- Network Size $(N)$ : The largest the network is in terms of peer population, the longer the dissemination takes to be accomplished. It is also critical to establish out how well the different protocols scale as the network size increases.

- Content Size (S): Flash dissemination in unstructured networks usually refers to medium-sized data (of $50 K B$ or less). Nevertheless it may refer to the rapid dissemination of image data captured by security cameras or to the fast spreading of alerts. Hence, it is crucial to determine which protocols work best for different content sizes.

Next, we briefly outline results from our experimentation while focusing on the above-mentioned four metrics.

Completion-Time (CT): In order to ascertain how the protocols efficiently scale in large $P 2 P$-networks we carried out experiments to assess the completion time of each protocol under varying content sizes for the communicated resources. Figure 4 depicts the behavior of Simple, Crew and Catalogue protocols in terms of $C T$ under varying peer population assessing their applicability for flash dissemination. Simple-Gossip has the worst performance among the

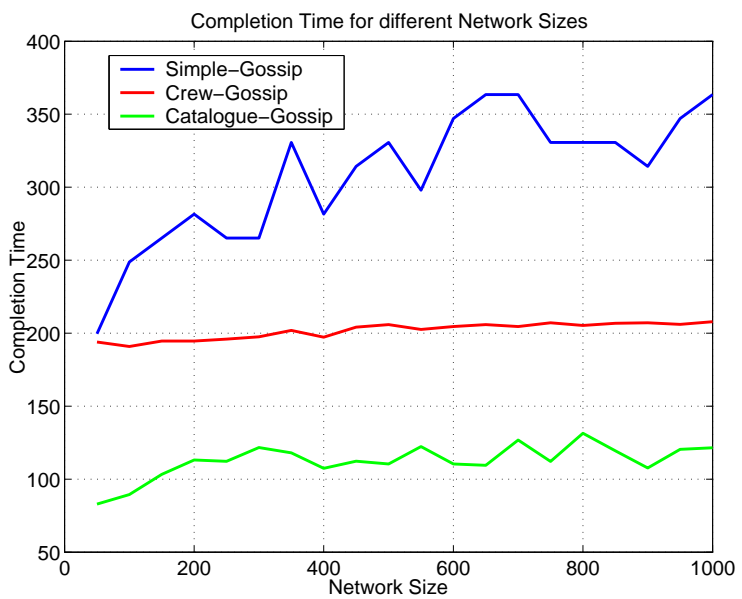

Figure 4. $C T s$ for different network sizes.

three protocols. It takes longer to disseminate the data in every case and consequently appears to scale poorly with increasing network size. This is not the case with Crew and Catalogue-Gossip which show more robust behavior in large-scale networks. Catalogue-Gossip however achieves better completion times as it completes in about half the time it takes for Crew-Gossip to do so. The same behavior appeared in our experiments even for extremely high number of nodes of up to $50 \mathrm{~K}$ peers where as Catalogue-Gossip still outperformed Crew.

Figure 5 depicts how the protocols react to different 
sizes of disseminated file for a 500-node network. We vary the size of the data resources from 5 to $50 \mathrm{~KB}$. Apparently, Simple-Gossip performs poorly whereas both Crew and Catalogue seem to scale roughly the same. We should mention here that the setting of this experiment favors Crew and is the worst case for Catalogue as disseminated data resources were split into a small number of chunks (five).

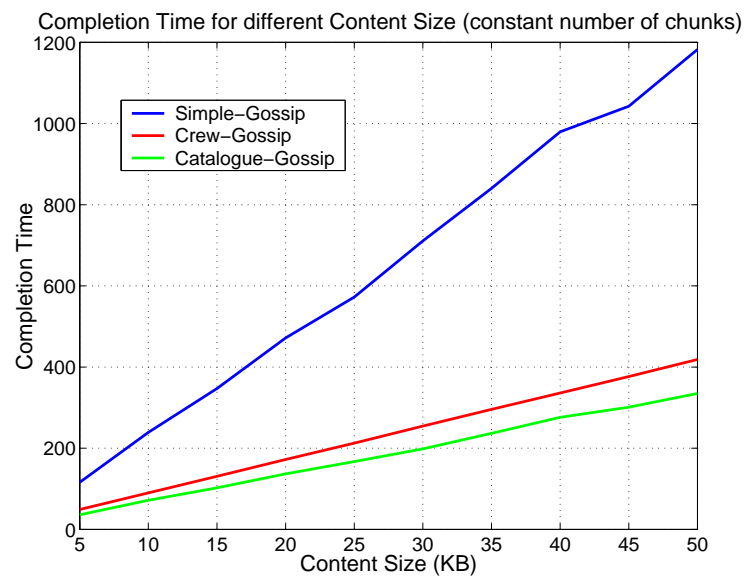

Figure 5. $C T$ s vs. varying content size.

Catalogue performs much better than Crew when resources are split in many chunks. For instance in the experimental setting of Figure 4, resources were fragmented into 10 chunks and the resulting performance of Catalogue was twice as good as Crew's. In cases of small chunks, the overhead of the numerous unsuccessful Crew pull requests increases and renders Crew inefficient when compared to Catalogue. Our proposed protocol scales well regardless of the splitting of resources into chunks and this further contributes to its robustness.

Coverage-Speed(CS): this measurement offers a view on how quickly a resource is disseminated throughout the network. Figure 6 depicts the rate of peer completion during the entire period of the dissemination for the three protocols. Although initially Simple closely follows Crew, it finally demonstrates much inferior rates as soon as about $80 \%$ of the peers have received the entire data resource, because the probability of selecting a random peer which has not completed yet falls dramatically. Moreover, Catalogue completes much earlier than Crew. This is because in Crew, the probability of selecting a peer with available chunks during the first steps of the dissemination is very small. This means that there is a significant delay in spreading the chunks in the network at the beginning of the dissemination, a fact reflected by the Crew curve in Figure 6.

Data-Overhead(DO): The reason behind the good performance of Catalogue-Gossip is that it manages to reduce data overhead. The protocol's design has focused on avoid-

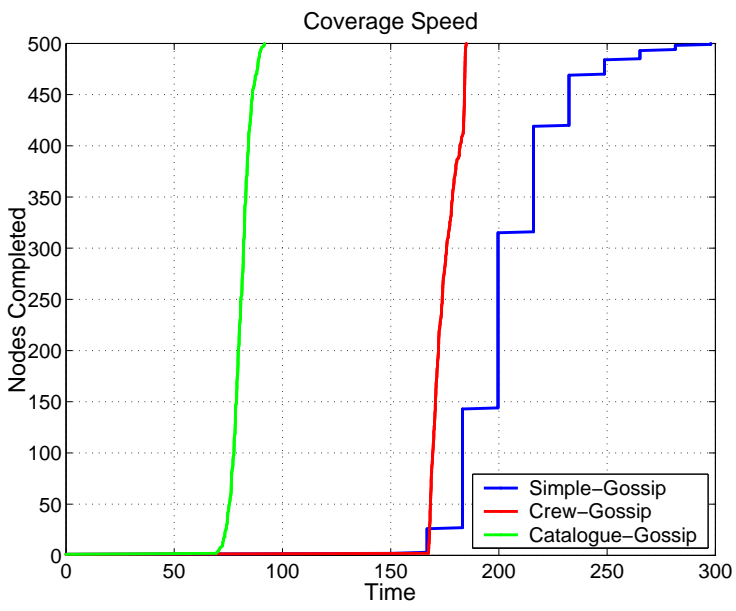

Figure 6. $C S$ s for the three protocols.

ing redundant transfers and unsuccessful messages. This section confirms that Catalogue-Gossip is more efficient in peer communication. Before continuing, it is useful to define what an "unsuccessful" message means in each protocol. First of all, an unsuccessful message in Simple-Gossip is a transfer of a duplicate chunk. As already analyzed, duplicate chunk transfers in Simple-Gossip are the source of high data overhead. In Crew-Gossip, an unsuccessful message is a pull request to a peer which has no chunk that is of interest to the requesting peer. Unsuccessful messages in Catalogue-Gossip reflect the event of a peer pushing its Catalogue to another node, which has all the chunks in the Catalogue. Figure 7 illustrates the number CatalogueGossip has about $80 \%$ less redundant messages than Crew). of redundant messages for the three protocols. CatalogueGossip has the smallest redundancy, an order of magnitude less than in Crew-Gossip (the vertical axis is logarithmic).

Chunk-Replication(CR): Chunk-replication examines how well chunk selection policies do in the context of Catalogue-Gossip. Figure 8 displays all three chunk selection policies evaluated in this paper, namely Random-First, Centralized Rarest-First and Decentralized Rarest-First policy. As Figure 8 shows, the centralized Rarest-First replicates the chunks of the original file the fastest in the system. This is because every peer selects the optimal chunk to fetch, as if there was a global state maintained about the frequency of chunks in the $P 2 P$ network. On the contrary, the Random-First policy takes much longer to achieve the same degree of replication. Moreover, difference between the above two policies becomes wider when it comes to files consisting of more chunks (Figure 8 refers to a resource of 10 chunks). This is because the expected number of random samples needed to draw all $N$ chunks increases rapidly with $N$. The Decentralized Rarest-First is a much better approximation to its centralized version, than 


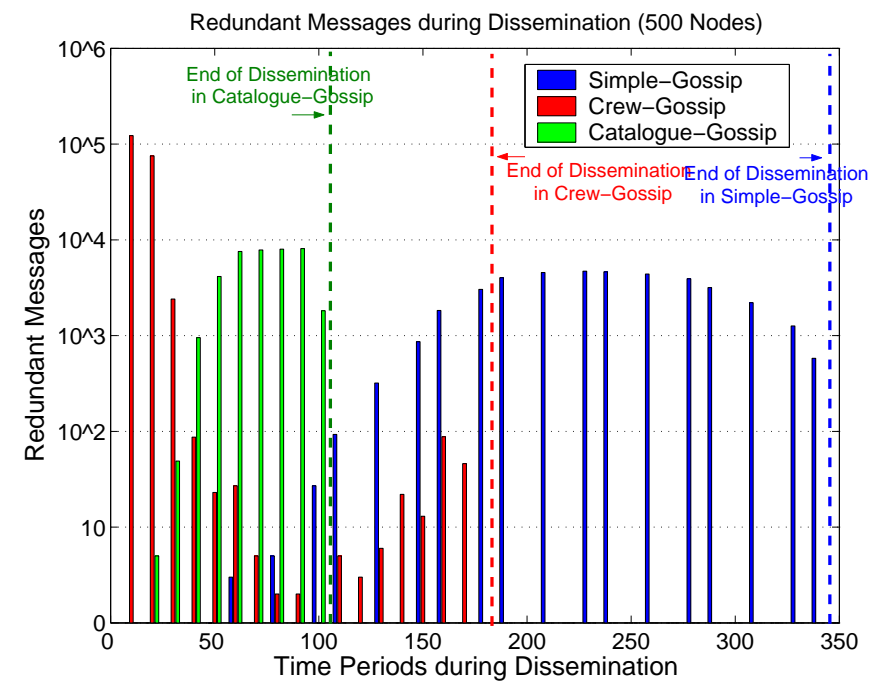

Figure 7. Redundant messages in SimpleGossip, Crew-Gossip and Catalogue-Gossip.

the naive Random-First policy.

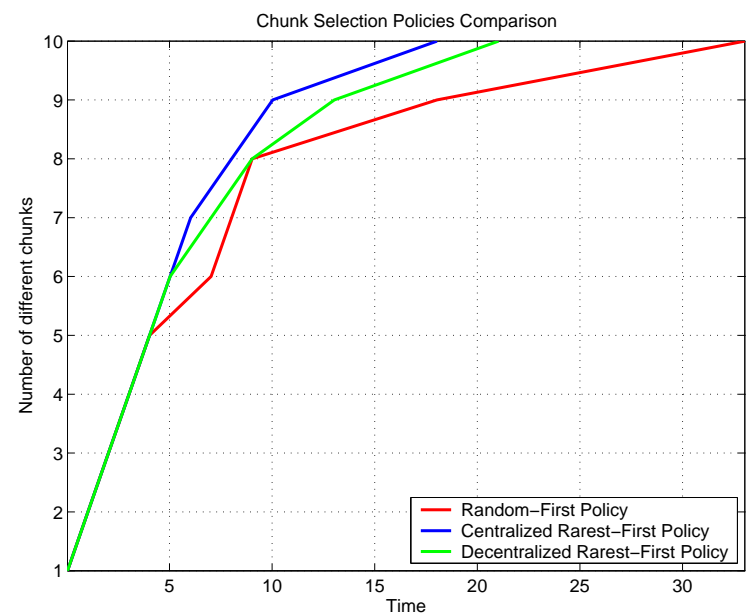

Figure 8. Time required by initiator to send all 10 chunks to other peers.

\section{Conclusions and Future Work}

In this paper, we proposed the Catalogue-Gossip protocol for addressing the problem of flash data dissemination in unstructured P2P-networks. Catalogue-Gossip experimentally shows improved performance behavior over existing counterparts as it features reduced data overheads and accelerated speed with which data reach their desti- nation nodes during the course of the dissemination process. Catalogue-Gossip also enforces deterministic delivery which is a strong requirement for data dissemination systems. Finally, Catalogue-Gossip implements a decentralized policy ensuring short time periods elapsed between initiation of a dissemination and the full replication of the data throughout the unstructured network. We plan to enhance the protocol by incorporating compression techniques to further reduce the volume of traded-data chunks and by exploiting specialized Bloom-filters to more efficiently represent protocol structures. We also intend to pursue an analytical approach in establishing bounds of feasible performance gains with respect to prior approaches.

\section{References}

[1] R. Barr, Z. J. Haas, and R. van Renesse. JiST: an Efficient Approach to Simulation using Virtual Machines. Software: Practice and Experience, 35(6):539-576, May 2005.

[2] B. Cohen. Incentives Build Robustness in BitTorrent. In Workshop on the Economics of Peer-to-Peer Systems, Berkeley, CA, June 2003.

[3] A. Demers, D. Greene, C. Hauser, W. Irish, J. Larson, S. Shenker, H. Sturgis, D. Swinehart, and D. Terry. Epidemic Algorithms for Replicated Database Maintenance. In 6th ACM Symposium on Principles of Distributed Computing, Vancouver, Canada, 1987.

[4] M. Deshpande, B. Xing, I. Lazaridis, B. Hore, N. Venkatasubramanian, and S. Mehrotra. CREW: A Gossip-based Flash-Dissemination System. In 26th IEEE Int. Conf. on Distributed Computing Systems, 2006.

[5] P. T. Eugster, R. Guerraoui, A.-M. Kermarrec, and L. Massoulie. Epidemic Information Dissemination in Distributed Systems. IEEE Computer, 37(5):60-67, May 2004.

[6] A. J. Ganesh, A.-M. Kermarrec, and L. Massoulie. Peer-to-Peer Membership Management for Gossip-based Protocols. IEEE Transactions on Computers, 52(2):139-149, February 2003.

[7] M. Jelasity, S. Voulgaris, R. Guerraoui, A.-M. Kermarrec, and M. van Steen. Gossip-based Peer Sampling. ACM Transactions on Computer Systems, 25(3):8, 2007.

[8] A.-M. Kermarrec, L. Masoulie, and A. J. Ganesh. Probabilistic Reliable Dissemination in Large-Scale Systems. IEEE Transactions on Parallel and Distributed Systems, 14(3):248-258, March 2003.

[9] K. Ramamritham and P. Shenoy. Dynamic Information Dissemination. IEEE Internet Computing, 11(4):14-15, 2007.

[10] R.Rivest. The MD5 Message-Digest Algorithm. RFC1321, 1992.

[11] S. Voulgaris and M. van Steen. An Epidemic Protocol for Managing Routing Tables in Very Large Peer-to-peer Networks. In 14th IFIP/IEEE Int. Workshop on Distributed Systems: Operations and Management (DSOM'03), 2003.

[12] A. Yu and S. T. Vuong. MOPAR: a Mobile Peer-to-Peer Overlay Architecture for Interest Management of Massively Multiplayer Online Games. In Int. Workshop on Network and Operating Systems Support For Digital Audio and Video (NOSSDAV'05), Stevenson, WA, 2005. 